\title{
Fronts between Rhythms: Spatiotemporal Dynamics of Extended Polyrhythmic Media
}

\author{
Julyan H.E. Cartwright, ${ }^{1}$ Raúl Montagne, ${ }^{2}$ Nicolás Piro, ${ }^{3}$ and Oreste Piro ${ }^{4,5}$ \\ ${ }^{1}$ Laboratorio de Estudios Cristalográficos, CSIC, E-18100 Armilla, Granada, Spain \\ ${ }^{2}$ Departamento de Fisica Teorica e Experimental, UFRN, Natal, Brazil \\ ${ }^{3}$ Institut de Ciéncies Fotóniques, Mediterranean Technology Park, 08860 Castelldefels, Barcelona, Spain \\ ${ }^{4}$ Institut Mediterrani d'Estudis Avançats, CSIC-UIB, E-07071 Palma de Mallorca, Spain \\ ${ }^{5}$ Center for Studies in Physics and Biology, Rockefeller University, New York, New York 10021, USA
}

(Received 22 February 2007; published 26 October 2007)

\begin{abstract}
We study the propagation of fronts in extended oscillatory reaction-diffusion systems that contain several coexisting limit cycles. In contrast with the variational behavior, fronts between regions oscillating in two different limit cycles are found to propagate not necessarily towards the region of the less stable limit cycle, but towards the regions of the largest amplitudes, provided that the frequency mismatch between the cycles is sufficiently large. In other words, the smaller oscillations can always be made to control the whole system.
\end{abstract}

DOI: 10.1103/PhysRevLett.99.174101

PACS numbers: $05.45 . \mathrm{Gg}$

Reaction-diffusion systems in which the reaction terms are oscillatory are ubiquitous in nature, not only in physics, chemistry, and biology, but also in economics and sociology [1-3]. Every living organism contains perhaps hundreds of chemical oscillators that operate in extended media where diffusion couples neighboring spatial points [4]. Weakly nonlinear oscillations in such an extended continuous medium can be described by an amplitude equation, the complex Ginzburg-Landau equation [5]. Less known, but perhaps equally widespread, is the class of systems in which oscillations may occur with more than one amplitude, or, to be more technical, in which the reaction term admits two or more stable limit cycles to which the dynamics may approach depending on the initial conditions [6-8].

Understanding the behavior of this neglected class of extended dynamical systems poses the following intriguing puzzle. When oscillators operating at different frequencies interact, synchronization sets in [9]. Generically, an asymptotic regime in which the fastest oscillators set the pace of the whole assembly is reached. If the interaction is diffusive, this phenomenon can be understood as the propagation of a front separating regions of fast and slow oscillations. Front propagation proceeds from regions populated by faster oscillators towards those of slower ones [2]; metaphorically speaking, the fastest wins. On the other hand, in variational reaction-diffusion systems such as the Fisher-Kolmogorov model [10] and others [2], fronts propagate between two locally stable states always in the direction of the less stable state [11], the metastable one in the case of a two-stable-state system. How far this behavior extends into the class of nonvariational systems is still a matter of debate, but it is widely accepted that if the system displays a Lyapunov stability function, this is a good indicator of the direction of the fronts: they travel in the direction of the less stable state. However, if so, this tendency conflicts with the fastest wins behavior of fronts between oscillating regions: one could conceive of situations where the most stable limit cycle is the slowest.

The purpose of this Letter is to investigate this conflict. We show that, in fact, neither of the two criteria of front propagation is valid for multiple-limit-cycle reactions. Instead, we find a tendency for the system to be dominated by the oscillators operating at the smallest amplitude, provided that their frequencies are different enough, but regardless of the sign of the difference. We construct a simple one-spatial-dimensional example to show this behavior, then we extend the results to a dirhythmic reactiondiffusion medium in two dimensions.

The simplest way of writing a reaction model having two different limit cycles is in polar coordinates:

$$
\begin{gathered}
\dot{R}=F(R)=-V^{\prime}(R), \\
\dot{\Theta}=\Omega(R),
\end{gathered}
$$

with, for example, the particular choice of

$$
V(R)=-\left\{\frac{\mu}{2}-\left[\frac{\varepsilon}{4}-\left(\frac{\gamma}{6}-\frac{\delta}{8} R^{2}\right) R^{2}\right] R^{2}\right\} R^{2}
$$

giving a limit cycle for each of the zeros of $F(R)$, the extrema of $V(R)$. The parameters $\mu, \varepsilon, \gamma$, and $\delta$ control the stability and amplitude of the limit cycles. In particular, the stable limit cycles are at the bottom of the "potential" wells, i.e., the relative minima $R_{1}$ and $R_{2}\left(R_{1}<R_{2}\right)$ of the function $V(R)$. Figure 1 shows $V(R)$ for several values of $\mu$, illustrating the fact that the relative height — and therefore the stability - of the $R_{1}$ and $R_{2}$ minima changes as this parameter is varied. The frequency of these limit cycles can be controlled independently by adopting different forms for the function $\Omega(R)$. Thus an increasing $\Omega(R)$ makes the limit cycle of radius $R_{2}$ oscillate at a frequency higher than that of the limit cycle of radius $R_{1}$, and vice versa. 


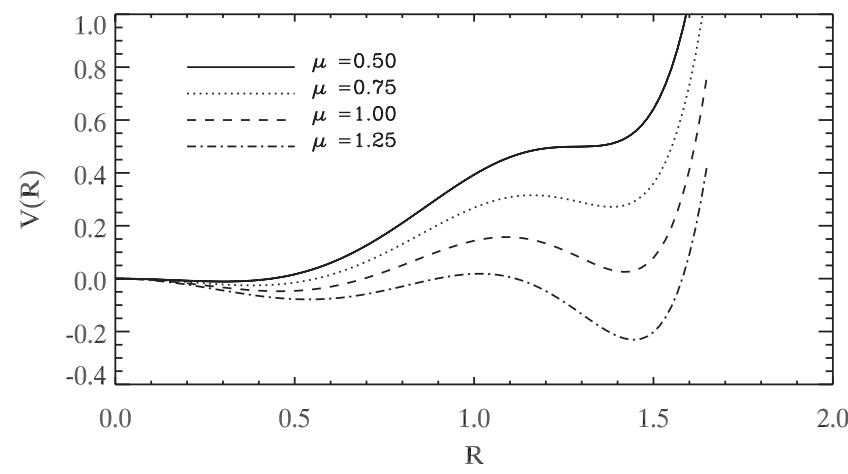

FIG. 1. Radial potential function $V(R)$ for several values of the parameter $\mu$. We fix $\varepsilon=6.12, \gamma=6.8208$, and $\delta=2.0$.

In order to include spatiotemporal dynamics, it is convenient to cast the previous equations into their Cartesian form by setting $X=R \cos \Theta$ and $Y=R \sin \Theta$. Initially, we are interested in the dynamics in one spatial dimension, so that $R=R(s, t), \Theta=\Theta(s, t), X=X(s, t)$, and $Y=Y(s, t)$ are all functions of the unique spatial coordinate $s$ and time. We consider here a purely diffusive spatial coupling isotropic in the Cartesian phase-space representation, with diffusion constant $D$. The complete model then reads

$$
\begin{gathered}
\dot{X}=f(R) X-\Omega(R) Y+D \partial_{s s} X, \\
\dot{Y}=f(R) Y+\Omega(R) X+D \partial_{s s} Y,
\end{gathered}
$$

with $f(R)=\mu-\left[\varepsilon-\left(\gamma-\delta R^{2}\right) R^{2}\right] R^{2} \quad$ and $\quad R^{2}=$ $X^{2}+Y^{2}$, supplemented with periodic boundary conditions without compromising generality.

We now investigate the evolution of initial field configurations in which one region is oscillating in one of the limit cycles and the rest of the domain in the other. We chose initial conditions of the form $R(s, 0)=0.5+\chi_{\left[s_{1}, s_{2}\right]}(s)$, where $\chi_{\left[s_{1}, s_{2}\right]}$ is the indicator function of the interval $\left[s_{1}, s_{2}\right]$. We first consider $\Omega(R)$ constant; the oscillation frequency is uniform across the phase space. As might be expected, this case reproduces the behavior of variational fronts in one spatial dimension: fronts separate regions in which the system is in each of two different locally stable static equilibria (potential minima), and they propagate in the direction of less stable ones, so that asymptotically the most stable stationary state dominates. Figure 2 shows that this is so also when, instead of fronts between two static equilibria, we consider those separating regions uniformly evolving in each of two different-but in this case, synchronous - oscillatory regimes. Fronts propagate towards the region where oscillations occur on the limit cycle that is less stable, in the sense that the radial potential is higher. This happens no matter what the relative amplitudes of the two are: the more stable always wins.

However, the situation changes in a rather surprising way when the frequencies of the two limit cycles are different, when $\Omega(R)$ is an arbitrary function. If the potential is such that the smaller limit cycle is the more stable, as
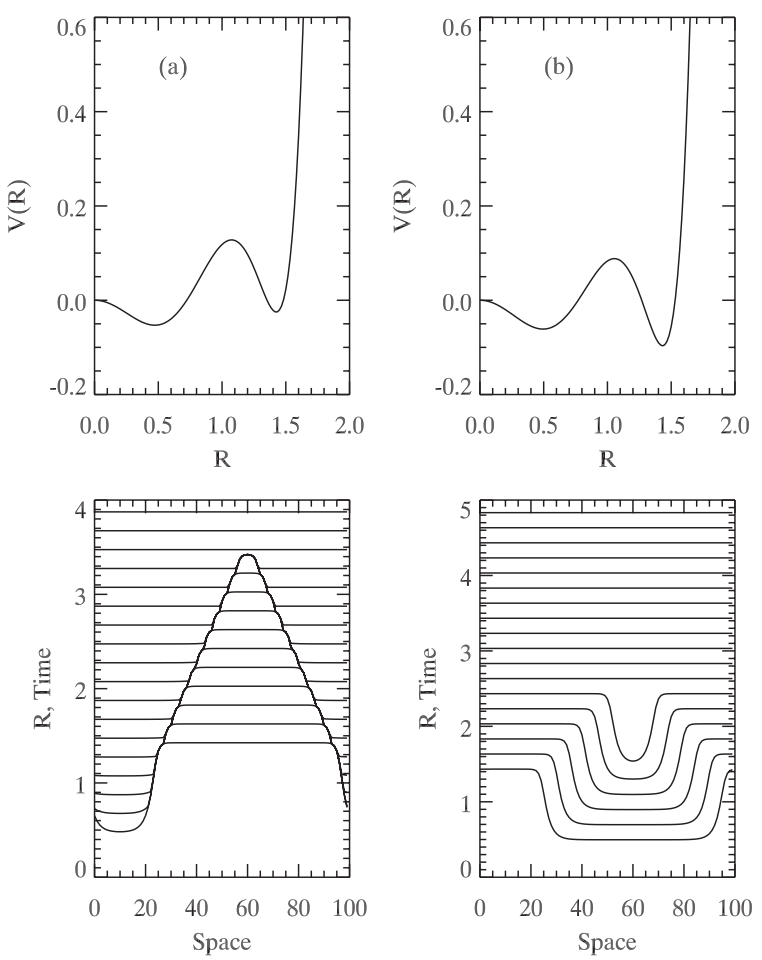

FIG. 2. Pseudo-3D space-time plots of $R(s, t)$ (bottom) showing fronts moving always towards the less stable minimum of the corresponding potential (top) for (a) $\mu=1.05$ and (b) $\mu=1.12$. $\Omega(R)=1$ and $D=0.1$. Space is in units of $\Delta s=0.1$ and the vertical axis labels in the bottom panels are the $R$ scale. Successive curves correspond to time increments of 15 and are raised by 0.2 with respect to the preceding curve.

in the case of Fig. 2(a), the fronts still move in the direction prescribed by the variational theory and as in the synchronous case. But if the more stable limit cycle is the larger, as in Fig. 2(b), when the difference $\Delta \Omega$ between the two limit-cycle frequencies exceeds a certain value, the fronts no longer propagate towards the region oscillating in the less stable limit cycle, but instead towards the regions oscillating with the largest amplitude. In short, the smallest can always win.

To illustrate this we set $\Omega(R)=\alpha \pm \beta R^{2}$ in Eqs. (4) and (5) and simulate the evolution of the same type of initial conditions as before. Figure 3 displays three different spatiotemporal evolutions obtained with the same potential [Fig. 3(a)] but with different values of $\beta$. While for small $\beta$ [Fig. 3(b)] the fronts progress towards the less stable inner limit cycle, by making $\beta$, and therefore $\Delta \Omega$, large enough, the propagation is reversed [Fig. 3(c)]. This reversal only depends on the size of $\beta$ or $\Delta \Omega$ but not on its sign, i.e., not on which of the two oscillators is faster; Fig. 3 remains essentially invariant under the change $\beta \rightarrow-\beta$. Thus, regardless of its stability, the inner limit cycle can always be made to asymptotically dominate the whole system by setting its frequency sufficiently different from the other's. 

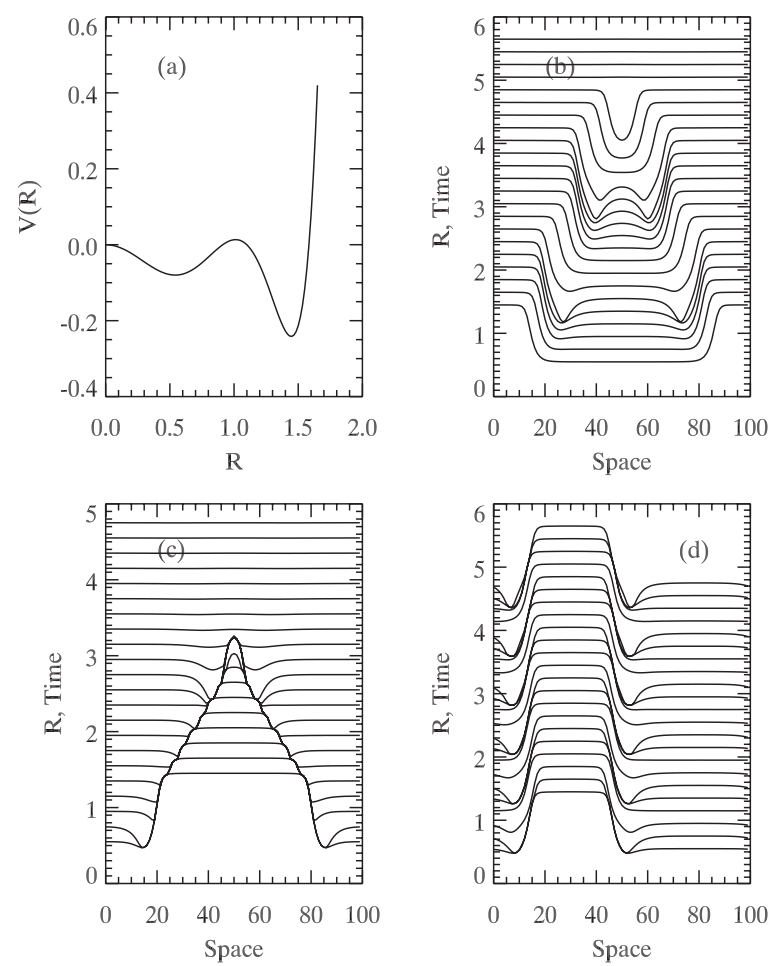

FIG. 3. (a) $V(R)$ for $\mu=1.26$. (b) The more stable limit cycle (the outer one) propagating over the less stable inner one for $\beta=$ 0.2. (c) Reversed propagation for $\beta=0.7$. (d) Coexistence of both limit cycles in a localized breathing structure for $\beta=0.35$.

To understand this behavior in a semianalytical way, let us recast Eqs. (4) and (5) back into polar coordinates:

$$
\begin{gathered}
\dot{R}=-V^{\prime}(R)-D\left(\partial_{s} \Theta\right)^{2} R+D \partial_{s s} R, \\
\dot{\Theta}=\Omega(R)+\frac{2 D}{R} \partial_{s} R \partial_{s} \Theta+D \partial_{s s} \Theta .
\end{gathered}
$$

The factor $R$ of the term $D\left(\partial_{s} \Theta\right)^{2} R$ from the spatial coupling can be regarded as an effective subtractive renormalization of the coefficient $\mu$ in the potential. Because of its sign, this renormalization can only make the largest limit cycle less stable. It is clear that this factor can play a role solely if the two limit cycles run at different frequencies. By taking the $s$ derivative of Eq. (7) we obtain an equation for the evolution of the phase gradient $G=\partial_{s} \Theta$ :

$$
\begin{aligned}
\dot{G}= & \Omega^{\prime}(R) \partial_{s} R-\frac{2 D}{R}\left[\frac{1}{R}\left(\partial_{s} R\right)^{2}-\partial_{s s} R\right] G \\
& +\frac{2 D}{R} \partial_{s} R \partial_{s} G+D \partial_{s s} G .
\end{aligned}
$$

The only source of $G$ is the term $\Omega^{\prime}(R) \partial_{s} R$ that vanishes if either $\Omega$ or $R$ are constant. As a consequence, gradient production is concentrated in the front region and is essentially proportional to the frequency jump across the front. This explains front propagation reversal in favor of the small-amplitude limit cycle when the frequency mismatch is large enough to produce a sufficiently large phase gradient as a qualitative extension of the variational results to the effective potential. The remaining terms in Eq. (8) stabilize the growth of $G$ by either dissipating, advecting, or diffusing the phase gradient.

We should like to mention here one interesting consequence of the peculiar topology of these fronts. While in the case of variational fronts between static equilibria, structures formed by complementary fronts either grow without limit or collapse except at a single critical value of the relative stability, dirhythmic media allow for sustained localized breathing structures that are stable for a finite range of values of either the frequency split or the parameters that control the relative stability of the limit cycles. In Fig. 3(d) we show one such structure in which a wall of medium oscillating in one limit cycle is separating two regions oscillating in the other cycle. With $\mu=1.26$, such structures are formed for $2.5 \lessgtr \beta \lesssim 6$.

We now investigate front propagation in dirhythmic reaction-diffusion media in two spatial dimensions. Let us consider the evolution of a circular patch of medium in one of the rhythmic states, surrounded by material oscillating at the other rhythm. In the analogous variational case of a medium that can be found in two different locally stable static equilibria, circular patches of the stable steady state surrounded by medium in the metastable state will grow only if their radii are larger than a critical value that depends solely on the potential energy difference between the two states, and metastable drops in a stable background always collapse. In dirhythmic media, however, the stabilities of the two oscillatory states may change, as we have seen, as a result of the phase dynamics. Equations (6) and (7) have the straightforward two-dimensional generalization

$$
\begin{aligned}
\dot{R} & =-V^{\prime}(R)-D|\nabla \Theta|^{2} R+D \nabla^{2} R, \\
\dot{\Theta} & =\Omega(R)+\frac{2 D}{R} \nabla R \cdot \nabla \Theta+D \nabla^{2} \Theta,
\end{aligned}
$$

where $X, Y, R, \Theta$ are now functions of the spatial coordinates $x$ and $y$ and time, and $\nabla$ and $\nabla^{2}$ are the twodimensional gradient and Laplacian operators. The second term in Eq. (9) can still be seen as an effective quadratic contribution to the potential $V(R)$ that tends to raise the local minimum corresponding to the larger limit cycle with respect to the smaller one. The consequences of this in two dimensions are slightly more complex than in one. Of course, a direct extension of the one-dimensional results is that patches of medium oscillating in the small limit cycle can always be forced to nucleate by controlling the frequency difference between the two cycles irrespective of the original relative stability. Moreover, since nucleation only occurs if the initial patch radius exceeds a critical value that depends on the relative stability of the asymptotic states, one can conjecture that for dirhythmic media the critical radius should depend on the frequency split in a qualitatively similar way.

In order to simulate the evolution of radially symmetric configurations, Eqs. (9) and (10) can be simplified by using 


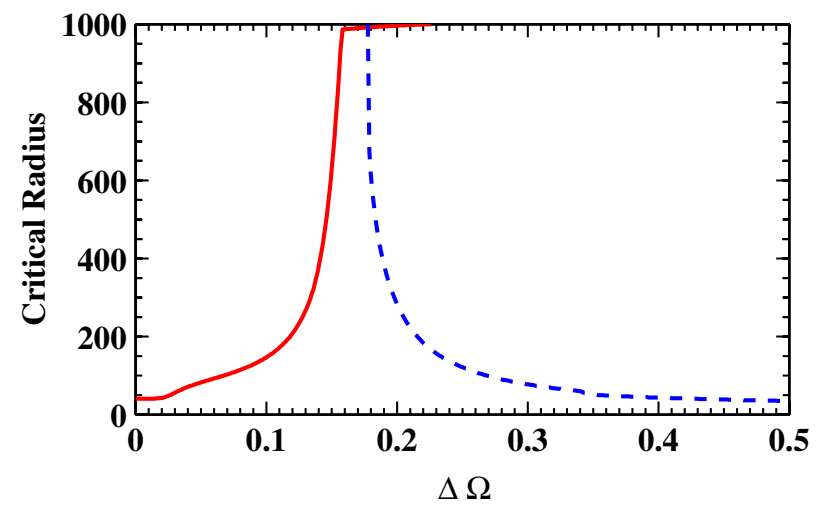

FIG. 4 (color online). Critical radius measured in units of $\delta r=0.05$ for the coalescence of a circular patch of medium oscillating in one of the limit cycles surrounded by medium oscillating in the other as a function of the frequency split $\Delta \Omega$. Solid and dashed curves correspond to patches of the large and small-amplitude oscillation, respectively.

polar coordinates $(r, \theta)$ and neglecting the angular variations:

$$
\begin{aligned}
& \dot{R}=-V^{\prime}(R)-D\left(\frac{\partial \Theta}{\partial r}\right)^{2} R+D\left(\frac{\partial^{2} R}{\partial r^{2}}+\frac{1}{r} \frac{\partial R}{\partial r}\right) \\
& \dot{\Theta}=\Omega(R)+\frac{2 D}{R} \frac{\partial R}{\partial r} \frac{\partial \Theta}{\partial r}+D\left(\frac{\partial^{2} \Theta}{\partial r^{2}}+\frac{1}{r} \frac{\partial \Theta}{\partial r}\right) .
\end{aligned}
$$

The only changes from Eqs. (6) and (7) are the curvature terms arising from the Laplacian. We impose von Neumann boundary conditions at $r=0$ to avoid singularities, and at the border of the circular domain to minimize the perturbation that this may exert on the dynamics of the patch. With these considerations in mind we can readily check our previous conjecture both qualitatively and quantitatively.

In Fig. 4 we summarize the results of the twodimensional dynamics by plotting the critical radius necessary for a circular patch of the medium oscillating in one of the limit cycles to grow against a background oscillating in the other limit cycle as a function of the frequency mismatch of the cycles. The potential is as in Fig. 2(b) so that the larger cycle is the more stable. The solid line corresponding to the critical radius for a patch of the large cycle diverges at around $\Delta \Omega=0.17$, which is a value close to that at which the reversal of the propagation would occur in one-dimensional systems. At the opposite extreme, in the limit of $\Delta \Omega \rightarrow 0$ this critical radius converges to the expected variational value. On the other hand, patches of the smaller inner cycle can grow only if $\Delta \Omega \gtrsim$ 0.17 with a critical radius monotonically decreasing with $\Delta \Omega$.

Fully two-dimensional simulations without radial symmetry confirm the validity of these results as well as the robustness of the one-dimensional walls described above. Remarkably, in two dimensions long-lived walls between head-on fronts with a structure similar to the breathers described in the one-dimensional case can form for a finite range of the frequency mismatch $\Delta \Omega$. These now represent bubbles that may either grow continuously or shrink and collapse. We provide movies [12] of the evolution of the bubbles, as well as of the spontaneous formation of onedimensional walls in two-dimensional systems starting from a randomly chosen initial condition.

The same behaviors reported in this Letter are seen in other polyrhythmic oscillators such as those described by generalized nonsymmetric van der Pol-Lienard equations with multiple limit cycles, which allows us to conjecture that they convey a high degree of universality.

This work was funded by Spanish MEC Grants No. CTQ2004-04648, No. FIS2005-07083-C02-02, sabbatical funds for O.P., CSIC project HIELOCRIS, and Brazilian agencies CNPq and FAPERN.

[1] G. Nicolis and I. Prigogine, Self-Organization in Nonequilibrium Systems, from Dissipative Structures to Order through Fluctuations (Wiley, New York, 1977).

[2] D. Walgraef, Spatio-Temporal Pattern Formation: With Examples from Physics, Chemistry, and Materials Science (Springer, New York, 1997).

[3] I. R. Epstein and J. A. Pojman, An Introduction to Nonlinear Chemical Dynamics: Oscillations, Waves, Patterns and Chaos (Oxford University Press, New York, 1998).

[4] Y. Kuramoto, Chemical Oscillations, Waves, and Turbulence (Springer, New York, 1984).

[5] I. Aranson and L. Kramer, Rev. Mod. Phys. 74, 99 (2002), and references therein.

[6] I. M. De la Fuente, L. Martinez, and J. Veguillas, BioSystems 35, 1 (1995); I. M. De la Fuente, BioSystems 50, 83 (1999).

[7] C. Pérez-Iratxeta et al., Biophys. Chem. 74, 197 (1998); A. Goldbeter et al., Chaos 11, 247 (2001).

[8] P. Bui, D. Vlachos, and P. Westmoreland, Combust. Flame 117, 307 (1999); T. Haberichter, M. Marhl, and R. Heinrich, Biophys. Chem. 90, 17 (2001).

[9] M. G. Rosenblum, A. S. Pikovsky, and J. Kurths, Synchronization: A Universal Concept in Nonlinear Sciences (Cambridge University Press, Cambridge, England, 2001).

[10] A. Kolmogorov, I. Petrovskii, and N. Piskunov, Bull. Univ. Moscou, Ser. Int., Sec. A 1, 1 (1937); translated in Dynamics of Curved Fronts, edited by P. Pelcé (Academic, New York, 1988); R. A. Fisher, Ann. Eugenics 7, 355 (1937).

[11] Y. Pomeau, Physica (Amsterdam) 23D, 3 (1986); P. Collet and J. Eckmann, Instabilities and Fronts in Extended Systems (Princeton University Press, Princeton, NJ, 1990); W. van Saarloos, Phys. Rep. 386, 29 (2003).

[12] See EPAPS Document No. E-PRLTAO-99-066743 for movies of the evolution of the 2D bubbles, as well the spontaneous formation of 1D walls in 2D systems starting from a randomly chosen initial condition. For more information on EPAPS, see http://www.aip.org/pubservs/ epaps.html. 